\title{
A FILAMENTARY STRUCTURE OF MASSIVE STAR-FORMING GALAXIES ASSOCIATED WITH AN X-RAY-ABSORBED QSO AT $z=1.8$
}

\author{
J. A. Stevens, ${ }^{1}$ M. J. Page, ${ }^{2}$ R. J. Ivison, ${ }^{1}$ Ian Smail, ${ }^{3}$ and F. J. Carrera ${ }^{4}$ \\ Received 2004 January 29; accepted 2004 February 3; published 2004 February 27
}

\begin{abstract}
The genesis of spheroids is central to our understanding of galaxy formation: they are relatively simple systems, containing about half the stellar mass of the universe. A major subset of spheroids, massive elliptical galaxies, are preferentially found in clusters where they exhibit old coeval stellar populations suggesting that they formed synchronously at early epochs. Here we report Submillimeter Common-User Bolometric Array submillimeter imaging of a region around a $z=1.8 \mathrm{X}$-ray-selected QSO. The image reveals a remarkable $\sim 400 \mathrm{kpc}$ long chain of galaxies, each with an obscured star formation rate sufficiently high to build a massive spheroid in less than 1 Gyr. The large overdensity of these galaxies relative to expectations for a random field implies that they probably reside in a structure associated with the QSO. We suggest that this star formation is associated with galaxy mergers or encounters within the filament, such as those predicted by the popular hierarchical model of galaxy formation. Our observations suggest that strong absorption in the X-ray spectra of QSOs at high redshifts may result from a veil of gas thrown up by a merger or merger-induced activity, rather than an orientationdependent obscuring torus. It is argued that these systems are the precursors of elliptical galaxies found today in the core regions of all rich galaxy clusters.
\end{abstract}

Subject headings: galaxies: evolution - galaxies: formation

On-line material: color figures

\section{INTRODUCTION}

Hierarchical structure formation models are predicated on the assumption that structures grow via the gravitational collapse of small inhomogeneities in a Gaussian random field. Within such a model it is expected that galaxies and larger scale structures will form at local maxima of the density field (Kaiser 1984). Inhomogeneities in such overdense regions, which will go on to become the most massive rich clusters at the present day, tend to collapse earlier than similar inhomogeneities in less-dense regions (Kauffmann 1996). Moreover, although the power spectrum of the fluctuations in these models is scale-free, detailed numerical simulations show that they naturally create large-scale filamentary structures with the most massive bound systems, such as rich clusters, forming at intersections and junctions of these filaments (Colberg et al. 1999). There is expected to be considerable cross talk between structures forming on different scales in these models, with galaxy halos growing through major mergers within the larger scale filaments and streaming down these into the forming cluster. These galaxies are most naturally identified as the progenitors of the homogeneous population of old luminous elliptical galaxies that reside in rich clusters at the present day. These models thus predict that the forming elliptical galaxies should be distributed in a highly anisotropic fashion around the collapsing protocluster (West 1994).

To test these predictions requires observations in a wave band sensitive to massive star formation at high redshifts. Recent progress in submillimeter astronomy has shown that star

\footnotetext{
${ }^{1}$ Astronomy Technology Centre, Royal Observatory, Blackford Hill, Edinburgh EH9 3HJ, UK; jas@roe.ac.uk, rji@roe.ac.uk.

${ }^{2}$ Mullard Space Science Laboratory, University College London, Holmbury St. Mary, Dorking, Surrey RH5 6NT, UK; mjp@mssl.ucl.ac.uk.

${ }^{3}$ Institute for Computational Cosmology, University of Durham, South Road, Durham DH1 3LE, UK; ian.smail@durham.ac.uk.

${ }^{4}$ Instituto de Fisica de Cantabria (Consejo Superior de Investigaciones Científicas-Universidad de Cantabria), Avenida de los Castros, 39005 Santander, Spain; carreraf@ifca.unican.es.
}

formation in massive galaxies at high redshifts is not a luminous phenomenon at optical wavelengths because of the obscuring effect of dust (Smail et al. 2002). The reprocessed starlight peaks at far-infrared wavelengths in the rest frame of the source and is shifted into the submillimeter wave band at $z>1$. We also require a method of identifying an overdense region of the early universe. One method is to target powerful active galactic nuclei (AGNs). Submillimeter images of the $\sim 1 \mathrm{Mpc}$ scale fields around such AGNs (high-redshift radio galaxies [HzRGs]) at $z>3$ show extended dust emission associated with the AGN and luminous submillimeter companions (Ivison et al. 2000; Stevens et al. 2003), several of which are confirmed to be at the same redshifts as the radio galaxies (Smail et al. 2003a, 2003b). While these results provide support for models of biased galaxy formation, they relate only to a very rare class of tracer galaxies, powerful HzRGs at $z>3$. Therefore, if we are to investigate the formation of structures the size of typical clusters, then less extreme, lower mass signpost AGNs at lower redshifts must be identified and their environments mapped at submillimeter wavelengths. With these points in mind we have initiated a program to image the fields around $1.5<z<3$ QSOs detected in the submillimeter wave band (Page et al. 2001). Here we report the first submillimeter imaging observations of one of these QSOs, RX J094144.51+385434.8 at $z=1.819$. A Hubble constant $H_{0}=70 \mathrm{~km} \mathrm{~s}^{-1} \mathrm{Mpc}^{-1}$ and density parameters $\Omega_{\Lambda}=0.7$ and $\Omega_{m}=0.3$ are assumed throughout this Letter.

\section{OBSERVATIONS AND DATA REDUCTION}

\subsection{Submillimeter Imaging}

Observations were made with the Submillimeter CommonUser Bolometric Array (SCUBA; Holland et al. 1999) at the James Clerk Maxwell Telescope in 2003 February and March. During all three nights on which data were gathered, weather conditions were in the top quartile of those experienced on Mauna Kea. We used "jiggle-map" mode to make simultaneous maps at 450 and $850 \mu \mathrm{m}$. The secondary mirror was chopped 

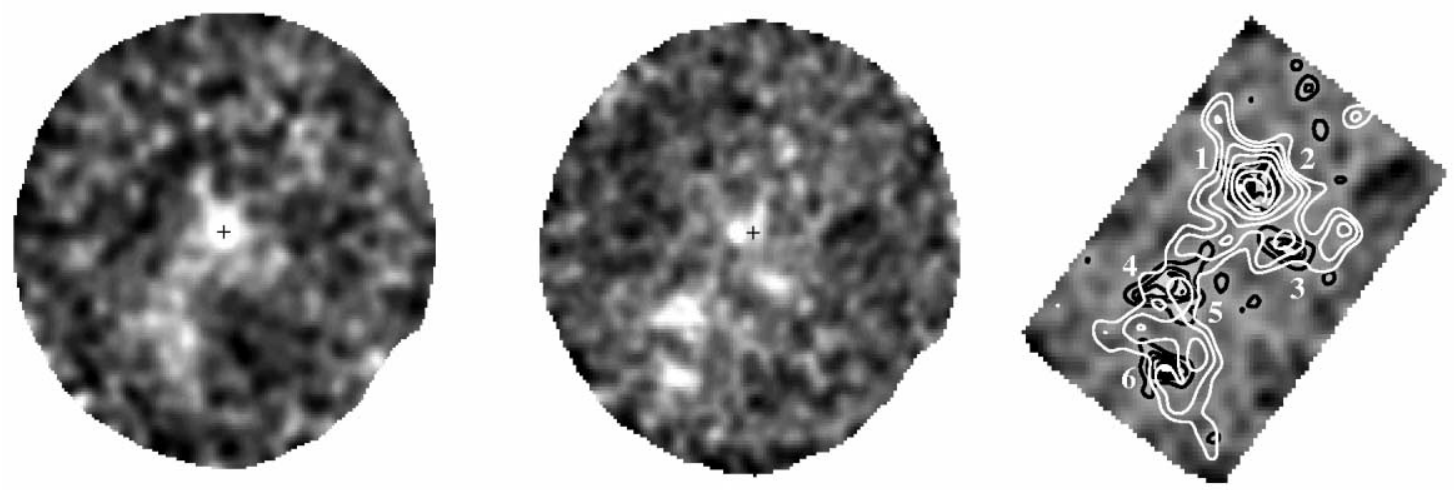

FIG. 1.-Submillimeter imaging of the field around RX J094144.51+385434.8. The left-hand panel shows the SCUBA $850 \mu \mathrm{m}$ image (diameter $\sim 150 "$, resolution 14".8). The middle panel shows the corresponding $450 \mu \mathrm{m}$ image (diameter $\sim 120^{\prime \prime}$, resolution 8".5). The right-hand panel shows a signal-to-noise ratio image $\left(1.5 \times 11^{\prime} 0\right)$ at $450 \mu \mathrm{m}$ (gray scale with black contours at 2,3, 4, and $\left.5 \sigma\right)$ overlaid with the $850 \mu \mathrm{m}$ signal-to-noise ratio contours at $2,3,4,5,6,7$, and $8 \sigma$. The extent of the contoured region is $\sim 400 \mathrm{kpc}$. Numbers on the right-hand panel identify $450 \mu \mathrm{m}$ sources with peak signal-to-noise ratio greater than 3 ; they correspond to those listed in Table 1. The optical position of the QSO is marked with a plus sign on the left-hand and middle panels. [See the electronic edition of the Journal for a color version of this figure.]

$45^{\prime \prime}$ in right ascension, and the antenna was nodded every $16 \mathrm{~s}$. The resulting maps thus show the negative off-positions of real sources (they have not been "cleaned"). The atmospheric opacity was monitored with regular skydips, and flux density calibration was made on each night with jiggle-map observations of the standard source CRL 618.

Data were analyzed with the STARLINK package, SURF. Data were first corrected for beam-switching, flat-fielded, and extinction-corrected. We then removed residual sky emission and clipped each bolometer at the $5 \sigma$ level before resampling the data onto an R.A./decl. grid and despiking at the $3 \sigma$ level. Pointing drifts were corrected for with linear interpolation between the measured offsets (in all cases less than 2"). After flux density calibration, the data were rebinned to make the final signal and noise maps. For this process we used an adapted version of the SURF task, REBIN. The standard deviation of the signal for each bolometer in each observation was first calculated, the data were then rebinned onto a 1" grid in R.A./ decl. coordinates, and the standard deviations were used to create weighted signal and weighted noise maps. These maps were subsequently smoothed with a $6^{\prime \prime}$ (at $850 \mu \mathrm{m}$ ) or $4^{\prime \prime}$ (at $450 \mu \mathrm{m})$ Gaussian.

\subsection{Optical and Near-Infrared Imaging}

An $R$-band image of a $16^{\prime} \times 16^{\prime}$ region centered on the QSO was taken at the $4.2 \mathrm{~m}$ William Herschel Telescope (WHT) using the Prime Focus Imaging Camera on the night of 2003 May 28. The total integration time was $3.7 \mathrm{ks}$, the seeing was 1.0 , and the conditions were photometric. The data were reduced using standard IRAF scripts and calibrated using Landolt faint standards (Landolt 1992). The $5 \sigma$ limiting magnitude for a point source in our 2".5 aperture is $R=25.4$.

A $K$-band exposure of the central $90^{\prime \prime} \times 90^{\prime \prime}$ part of the field was obtained in photometric conditions with the UKIRT FastTrack Imager on the United Kingdom Infrared Telescope (UKIRT) on 2003 May 24. The total exposure time was $7.7 \mathrm{ks}$ in 0.6 seeing, yielding a $5 \sigma$ limiting magnitude of $K=20.5$. The data were reduced using the ORAC-DR pipeline, and the resulting mosaics combined with STARLINK CCDPACK tasks. The data were calibrated with observations of the UKIRT faint standard FS 127 (Hawarden et al. 2001).

Galaxies were identified on the $K$-band frame using SExtractor (Bertin \& Arnouts 1996) and total magnitudes estimated from BEST_MAG. The positions were then used to measure colors within $2 " .5$ diameter apertures from the seeingmatched $R / K$ frames.

\section{RESULTS AND DISCUSSION}

The 450 and $850 \mu \mathrm{m}$ images reveal a remarkable structure of submillimeter sources in the field of the QSO, particularly striking at $450 \mu \mathrm{m}$ (Fig. 1). For the high-redshift $(z>3)$ objects targeted to date (Stevens et al. 2003), the slightly different negative $K$ -

TABLE 1

Source Names, Coordinates, Submillimeter Flux Densities, and Parameters

\begin{tabular}{|c|c|c|c|c|c|c|c|c|}
\hline Number $^{\mathrm{a}}$ & Source Name ${ }^{b}$ & R.A. $(\mathrm{J} 2000.0)^{\mathrm{c}}$ & Decl. $(\mathrm{J} 2000.0)^{\mathrm{c}}$ & $\begin{array}{c}S_{850} \\
(\mathrm{mJy})\end{array}$ & $\begin{array}{c}S_{450} \\
(\mathrm{mJy})\end{array}$ & $\begin{array}{l}\text { Dust Mass } \\
\qquad\left(M_{\odot}\right)\end{array}$ & $\begin{array}{l}L_{\mathrm{FIR}} \\
\left(L_{\odot}\right)\end{array}$ & $\begin{array}{c}\text { SFR } \\
\left(M_{\odot} \mathrm{yr}^{-1}\right)\end{array}$ \\
\hline 1 & RX J094144 SMM 1 & 094144.96 & +38 5439.0 & $13.4 \pm 1.5^{\mathrm{d}}$ & $44.3 \pm 7.6$ & $7.1 \times 10^{8}$ & $1.6 \times 10^{13}$ & 2800 \\
\hline 2 & RX J094144 SMM 2 & 094144.49 & +385442.0 & $13.4 \pm 1.5$ & $29.8 \pm 7.9$ & $4.8 \times 10^{8}$ & $1.1 \times 10^{13}$ & 1900 \\
\hline 3 & RX J094144 SMM 3 & 094144.00 & +385424.8 & $6.6 \pm 1.5$ & $32.6 \pm 8.3$ & $5.3 \times 10^{8}$ & $1.1 \times 10^{13}$ & 1900 \\
\hline 4 & RX J094144 SMM 4 & 094146.63 & +385415.8 & $8.0 \pm 1.3$ & $33.3 \pm 8.9$ & $5.4 \times 10^{8}$ & $1.2 \times 10^{13}$ & 2100 \\
\hline & RX J094144 SMM 5 & 094146.16 & +385416.0 & $8.0 \pm 1.3$ & $37.8 \pm 9.4$ & $6.1 \times 10^{8}$ & $1.3 \times 10^{13}$ & 2200 \\
\hline $6 \ldots \ldots$ & RX J094144 SMM 6 & 094146.48 & +385358.8 & $6.5 \pm 1.8$ & $39.7 \pm 8.6$ & $6.4 \times 10^{8}$ & $1.4 \times 10^{13}$ & 2400 \\
\hline
\end{tabular}

Note.-Units of right ascension are hours, minutes, and seconds, and units of declination are degrees, arcminutes, and arcseconds.

${ }^{\text {a }}$ Source numbers are those printed in Fig. 1.

${ }^{\mathrm{b}}$ Source names are based on the ROSAT R.A. position of the QSO

'Source coordinates are taken from the $450 \mu \mathrm{m}$ map that is centered on the QSO at R.A. $=09^{\mathrm{h}} 41^{\mathrm{m}} 44.51$, decl. $=+38^{\circ} 54^{\prime} 34^{\prime \prime} .8(\mathrm{~J} 2000.0)$. The formal uncertainties on these positions, given by $\mathrm{FWHM} /(\mathrm{S} / \mathrm{N} \times 2)$, are only $0.7-1{ }^{\prime \prime} .2$, although it should be assumed that systematic effects will at least double these values.

${ }^{\mathrm{d}}$ The QSO (cf. $10.1 \pm 1.7 \mathrm{mJy}$ from Page et al. 2001). 

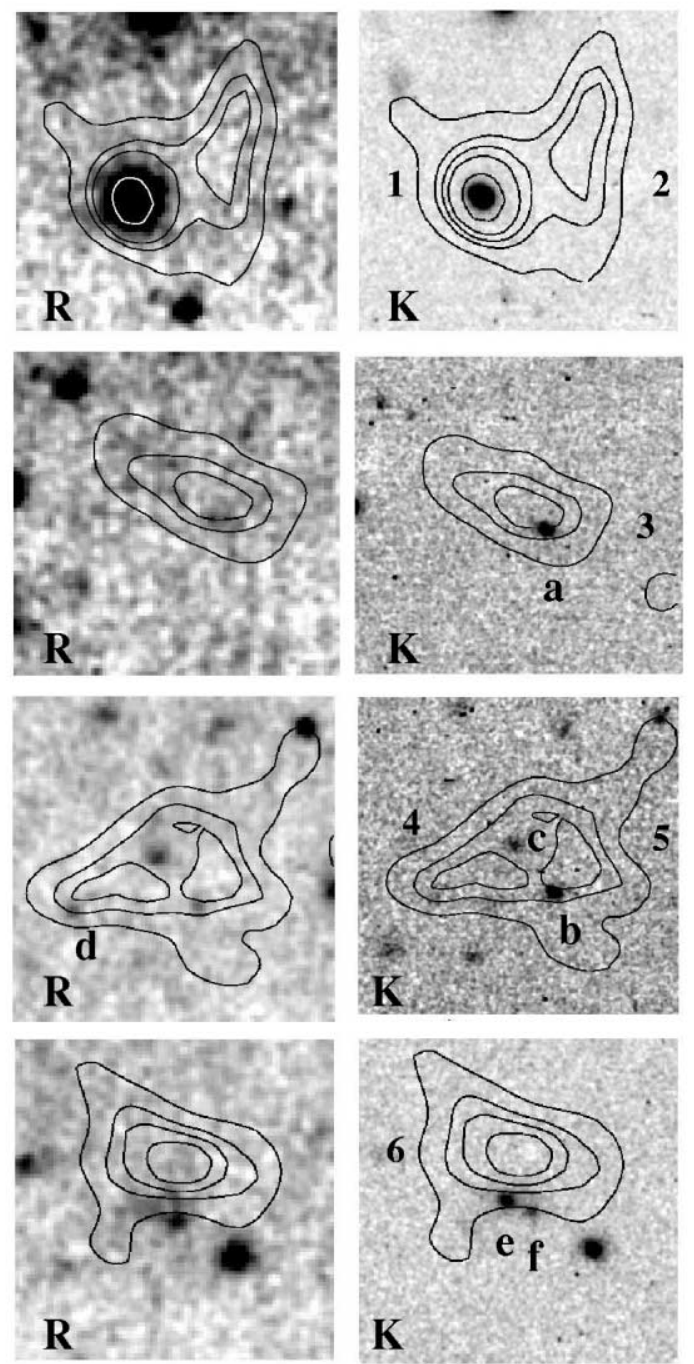

FIG. 2.-Optical and near-infrared imaging of the submillimeter galaxies in the vicinity of RX J094144.51+385434.8. The R.A./decl. images are $22^{\prime \prime}$ square, equivalent to $200 \mathrm{kpc}$ at $z=1.8$. Optical $R$-band images ( $1^{\prime \prime}$ seeing) from the WHT and near-infrared $K$-band images (0"6 seeing) from UKIRT overlaid with the $450 \mu \mathrm{m}$ contours from Fig. 1 (the $450 \mu \mathrm{m}$ image has been offset by $\left[-4.9^{\prime \prime},+0.3\right]$ to center the dust peak on the optical/near-infrared source). Numbers on the panels are those from Fig. 1 and Table 1. The magnitudes of these counterparts are (1) $K=19.3,(R-K)=5.8$, (2) $K=19.9$, $(R-K)=5.3,(3) K=20.3,(R-K)=3.9,(4) R=24.9,(R-K)<3.6$, (5) $K=18.8,(R-K)=5.4$, and (6) $K=19.5,(R-K)=4.6$. [See the electronic edition of the Journal for a color version of this figure.]

corrections that act on the 850 and $450 \mu \mathrm{m}$ flux densities have resulted in poor-quality $450 \mu \mathrm{m}$ images. This is because while the $850 \mu \mathrm{m}$ datum is shifted along the steep Rayleigh-Jeans tail of the dust emission, effectively canceling out the cosmological dimming, the $450 \mu \mathrm{m}$ datum is shifted close to the peak of this emission giving a marked falloff of the flux density with redshift. In this respect, the lower redshift of RX J094144.51+385434.8 has worked to our advantage, providing us with an image of the submillimeter dust emission with unprecedented resolution (8.5, equivalent to $\sim 70 \mathrm{kpc}$ at $z=1.8$ ).

We identify six sources with peak signal noise greater than 3 in the $450 \mu \mathrm{m}$ image; they trace a "chain" of submillimeter galaxies with an extent of at least $400 \mathrm{kpc}$ (if at $z=1.8$ ). Source names, coordinates, and physical quantities calculated from the images are presented in Table 1. At $450 \mu \mathrm{m}$ the sources are well enough separated to allow good estimation of their individual flux densities; this is not the case at $850 \mu \mathrm{m}$, where we give combined flux densities for numbers 1,2 (the QSO) and 4, 5. Quoted errors do not include the calibration uncertainties, which are about $10 \%$ at $850 \mu \mathrm{m}$ and $15 \%-20 \%$ at $450 \mu \mathrm{m}$. Dust masses are calculated from the $450 \mu \mathrm{m}$ flux densities in the standard manner (Hildebrand 1983), adopting a value for the dust mass absorption coefficient $\kappa_{100 \mu \mathrm{m}}=$ $5.5 \mathrm{~m}^{2} \mathrm{~kg}^{-1}$ (Draine \& Lee 1984). Far-infrared luminosities $\left(L_{\mathrm{FIR}}\right)$ are calculated by scaling the $450 \mu \mathrm{m}$ emission with that of Mrk 231, which has $L_{\mathrm{FIR}}=2.0 \times 10^{12} L_{\odot}$ (calculated from a graybody fit to the far-infrared millimeter spectral energy distribution; the fitted dust temperature is $42 \mathrm{~K}$ ). Using Arp 220 as a template reduces the $L_{\mathrm{FIR}}$ estimates by a factor of $\sim 1.6$. The star formation rates are calculated from $\operatorname{SFR}\left(M_{\odot} \mathrm{yr}^{-1}\right)=L_{\mathrm{FIR}} /$ $\left(5.8 \times 10^{9} L_{\odot}\right)($ Kennicutt 1998).

Each galaxy in this filament is itself a very massive ultraluminous infrared galaxy (ULIRG) producing stars at a rate sufficient to build a massive spheroid in less than 1 Gyr. Let us assess the significance of this structure. The density of $450 \mu \mathrm{m}$ sources in this field is $1.4 \pm 0.6 \mathrm{arcmin}^{-2}$, disregarding the QSO that was already known to be a submillimeter source. The corresponding density of sources found in "blank field" surveys with $450 \mu \mathrm{m}$ flux densities in excess of $30 \mathrm{mJy}$ is uncertain, but best estimates lie in the range $0.03-0.14 \mathrm{arcmin}^{-2}$ (Smail et al. 2002). These figures thus imply an order of magnitude overdensity of luminous star-forming galaxies in the $3.5 \operatorname{arcmin}^{2}$ field around RX J094144.51+385434.8. We can investigate the statistical significance of this overdensity by calculating the combined probability that these sources each exist at a distance, $d$, from the QSO given the expectation from the known surface density of blank field objects. The high end of the range given above, i.e., $n=0.14 \mathrm{arcmin}^{-2}$, gives the most pessimistic estimate of this probability [given by $P=$ $1-\exp \left(-\pi n d^{2}\right)$ for each source]. We find $P=2.9 \times 10^{-7}$ that the structure we observe is a chance superposition of field objects $\left(P=6.5 \times 10^{-5}\right.$ if source 2 is excluded $)$. This result provides very strong evidence that the companion galaxies lie at the same redshift and in the same structure as the QSO. Assuming this to be true, the star formation rate density calculated in a $500 \mathrm{kpc}$ cube centered on this structure is greater than $1000 M_{\odot} \mathrm{yr}^{-1} \mathrm{Mpc}^{-3}$, about 3-4 orders of magnitude higher than found in unbiased optical, infrared, and submillimeter surveys (Ivison et al. 2002; Chapman et al. 2003). This field thus provides a striking demonstration of the filamentary distribution of forming galaxies in a high-density region at high redshift.

Can we find further support for the hierarchical model in our data? In the high-resolution $450 \mu \mathrm{m}$ image, the central QSO and at least one of the spatially distinct submillimeter sources have complex morphologies, indicative of either merging or interacting systems. These characteristics are analogous to those observed in local ULIRGs (Joseph 1990), although these have more compact millimeter/submillimeter emission (Downes \& Solomon 1998) than observed here at higher redshift. Moreover, these mergers must be occurring between similarly massive (Table 1) and gas-rich systems, indicating that this activity is arising from major, rather than minor, mergers. Images of the RX J094144.51+385434.8 field in the $R$ and $K$ band are shown in Figure 2. The submillimeter galaxies RX J094144 SMM 3, RX J094144 SMM 4/5, and RX J094144 SMM 6 all have counterparts identified as extremely red objects (Elston, Rieke, \& Rieke 1988) — defined as having $(R-K)>5.3$ (Pozzetti \& Mannucci 2000). These objects are often found as counterparts to submillimeter galaxies discovered in blank field surveys (Smail et al. 1999; Ivison et al. 2002). The counterpart of RX 
J094144 SMM 6 is particularly interesting; it has the appearance of an advanced merger and is a blue/red composite source, again similar to many counterparts of blank field submillimeter galaxies (Ivison et al. 2002). Similar evidence for a merger origin of submillimeter galaxies has been seen in Chandra observations that show them to be coincident with two or three X-ray sources-interpreted as obscured AGNs buried in merging star-forming galaxies (Smail et al. 2003b; Alexander et al. 2003). If the submillimeter-detected galaxies are protoelliptical galaxies, then at $z \sim 2$ they should contain growing black holes that shine as AGNs (Kauffmann \& Haehnelt 2000; Page et al. 2001). However, the ROSAT data for this field show that the QSO is the only luminous X-ray source among them. If the other dusty galaxies contain AGNs, then they have to be of lower luminosity or more highly obscured or both. Observations with the new generation of X-ray telescopes will allow us to probe the evolutionary state of the black holes in these systems (Smail et al. 2003b).

These images are the first to be made of a high-redshift X-rayabsorbed QSO at submillimeter wavelengths. The importance of these objects relative to their better studied X-ray-unabsorbed (or optically selected) counterparts is only just becoming clear. For matched $(1<z<3)$ samples selected close to the break of the Xray luminosity function, the latter are about 1 order of magnitude less luminous at submillimeter wavelengths. The most likely interpretation is that the X-ray absorption in these objects is not linked with orientation-dependent obscuration-the "unified scheme" (Antonucci 1993) — but rather with the evolutionary state of the galaxy (Page et al. 2004). Our submillimeter images indicate that this is indeed the case for RX J094144.51+385434.8: its submillimeter luminosity appears to originate in large-scale star formation associated with a major galaxy merger.
Finally, let us consider the fate of this structure. Deep Hubble Space Telescope images of the fields around QSOs (McLure \& Dunlop 2001) suggest that their typical environments are galaxy clusters of Abell richness class 0 (Abell 1958). At the present day such clusters contain within their virial radius of the order of 10 galaxies with luminosity greater than or equal to $L^{*}$ (Christlein \& Zabludoff 2003). If the six submillimeter luminous galaxies in the protocluster around RX J094144.51+385434.8 continue to form stars at the rates inferred from their submillimeter luminosities (Table 1), then their transformation into $L^{*}$ elliptical galaxies will be complete within in a fraction of a gigayear. We can thus conclude that the coeval stellar populations of the luminous cluster galaxies will be in place by redshift 1.7 . Their subsequent passive luminosity evolution will naturally result in a population of elliptical galaxies with properties that match the core regions of today's rich galaxy clusters.

The James Clerk Maxwell Telescope is operated by the Joint Astronomy Centre in Hilo, Hawaii, on behalf of the parent organizations PPARC in the United Kingdom, the National Research Council of Canada, and the Netherlands Organization for Scientific Research. Near-infrared data were collected as part of the UKIRT Service Programme. UKIRT is operated by the Joint Astronomy Centre on behalf of PPARC. The WHT is operated on the island of La Palma by the Isaac Newton Group in the Spanish Observatorio del Roque de los $\mathrm{Mu}-$ chachos of the Instituto de Astrofisica de Canarias. J. A. S. acknowledges support from PPARC, I. S. from the Royal Society, and F. J. C. from the Spanish Ministerio de Cienca y Technología, under project AYA2000-1690.

\section{REFERENCES}

Abell, G. 1958, ApJS, 3, 211

Alexander, D. M., et al. 2003, AJ, 125, 383

Antonucci, R. 1993, ARA\&A, 31, 473

Bertin, E., \& Arnouts, S. 1996, A\&AS, 117, 393

Chapman, S. C., Blain, A. W., Smail, I., \& Ivison, R. J. 2003, Nature, 422, 695

Christlein, D., \& Zabludoff, A. I. 2003, ApJ, 591, 764

Colberg, J. M., White, S. D. M., Jenkins, A., \& Pearce, F. R. 1999, MNRAS, 308, 593

Downes, D., \& Solomon, P. M. 1998, ApJ, 507, 615

Draine, B. T., \& Lee, H. M. 1984, ApJ, 285, 89

Elston, R., Rieke, G. H., \& Rieke, M. J. 1988, ApJ, 331, L77

Hawarden, T. G., Leggett, S. K., Letawsky, M. B., Ballantyne, D. R., \& Casali, M. M. 2001, MNRAS, 325, 563

Hildebrand, R. D. 1983, QJRAS, 24, 267

Holland, W. S, et al. 1999, MNRAS, 303, 659

Ivison, R. J., et al. 2000, ApJ, 542, 27 2002, MNRAS, 337, 1

Joseph, R. D. 1990, in Dynamics and Interactions of Galaxies, ed. R. Wielen (New York: Springer), 132
Kaiser, N. 1984, ApJ, 284, L9

Kauffmann, G. 1996, MNRAS, 281, 487

Kauffmann, G., \& Haehnelt, M. 2000, MNRAS, 311, 576

Kennicutt, R. C., Jr. 1998, ApJ, 498, 541

Landolt, A. U. 1992, AJ, 104, 340

McLure, R. J., \& Dunlop, J. S. 2001, MNRAS, 321, 515

Page, M. J., Stevens, J. A., Ivison, R. J., \& Carrera, F. J. 2004, Science, submitted

Page, M. J., Stevens, J. A., Mittaz, J. P. D., \& Carrera, F. J. 2001, Science, 294, 2516

Pozzetti, L., \& Mannucci, F. 2000, MNRAS, 317, L17

Smail, I., Ivison, R. J., Blain, A. W., \& Kneib, J.-P. 2002, MNRAS, 331, 495

Smail, I., Ivison, R. J., Gilbank, D. G., Dunlop, J. S., Keel, W. C., Motohara, K., \& Stevens, J. A. 2003a, ApJ, 583, 551

Smail, I., Scharf, C. A., Ivison, R. J., Stevens, J. A., Bower, R. G., \& Dunlop, J. S. 2003b, ApJ, 599, 86

Smail, I., et al. 1999, MNRAS, 308, 1061

Stevens, J. A., et al. 2003, Nature, 425, 264

West, M. J. 1994, MNRAS, 268, 79 